\title{
A Maple Syrup Extract Prevents $\beta$-Amyloid Aggregation
}

\author{
Cassandra LA Hawco, YanFei Wang, Marcia Taylor, Donald F Weaver
}

Keywords: Alzheimer's, dementia

doi:10.1017/cjn.2015.270

Can J Neurol Sci. 2016; 43: 198-201

\section{BACKGROUND}

During our systematic search for natural product-based anti-protein misfolding agents for the putative treatment of Alzheimer's disease (AD), we investigated maple syrup. Canada is the world's largest producer of maple syrup, accounting for $80 \%$ of global output; 2013 annual production was 46 million litres. ${ }^{1}$ Maple syrup is produced by thermal evaporation of the sap from maple (Acer) trees, a process which concentrates the contained sugars as well as producing a variety of chemical reactions responsible for the distinct colour and taste of the syrup. In addition to the sugars (predominantly $\alpha$-D-glucopyranosyl$(1 \rightarrow 2)$ - $\beta$-D-fructofuranoside), the collected sap contains a wide range of naturally occurring oligosaccharides, amino acids, polyphenols and phytohormones. The prolonged high temperature thermal process involved in producing syrup from sap also results in the formation of a variety of other phenolic by-product compounds. Maple syrup extracts have been reported to exhibit a variety of health-related properties including antioxidant, antimutagenic and anti-cytoproliferative activities. ${ }^{2,3}$ In this study we demonstrate that an ethyl acetate maple syrup extract decreases oligomerization and aggregation of both $\beta$-amyloid $(\mathrm{A} \beta)$ and tau peptides in vitro, the two pathological hallmarks of AD.

\section{METHODS \\ Extractions}

Maple syrup (Canada No. 3 - Dark; White Meadows Farms, St. Catherine's, Ontario) was extracted following previously described procedures. ${ }^{5}$ One litre of syrup was first extracted with ethyl acetate $(3 \times 500 \mathrm{~mL})$ and dried using sodium sulfate prior to evaporation of the combined fractions in vacuo to yield $0.118 \mathrm{~g}$ of a light brown solid (Extract A). Further extraction of the syrup with $n$-butanol $(3 \times 500 \mathrm{~mL})$ yielded a brown solid following drying with sodium sulfate and removal of solvent in vacuo which was then extracted $(3 \times 10 \mathrm{~mL})$ to produce a methanol soluble (1.40 $\mathrm{g}$ brown oil) and insoluble (2.31 $\mathrm{g}$ light brown solid) extracts (Extracts $\mathbf{B}$ and $\mathbf{C}$ ). Previous studies by Li and Seeram, using the same extraction method, have characterized the components of the ethyl acetate and $n$-butanol extracts of maple syrup. ${ }^{4,5}$ Thirty different compounds including a variety of ligands and polyphenols were identified in the ethyl acetate extract, ${ }^{4}$ while the $n$-butanol extract contained an additional 23 compounds including lignans, phenolic derivatives, stillbene and coumarins. ${ }^{5}$ Solutions of the extracts were prepared in dimethylsulfoxide (DMSO) in various concentrations for in vitro testing, with tested concentrations of 30, 6 and $1.2 \mathrm{ppm}$.

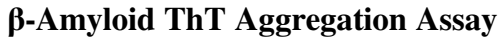

Thioflavin T (ThT; 4-(3,6-dimethyl-1,3-benzothiazol-3ium-2-yl)-N,N-dimethylaniline chloride) is a benzothiazole dye with a high affinity for proteins containing high $\beta$-sheet content used in the visualization of $\beta$-amyloid aggregates. Unbound ThT has fluorescence excitation ( $\lambda$ ex $)$ and emission ( $\lambda$ ex $)$ wavelengths at $430 \mathrm{~nm}$ and $342 \mathrm{~nm}$ respectively, which upon binding to aggregated $\beta$-amyloid undergoes a characteristic spectral shift $(\lambda \mathrm{ex}=442 \mathrm{~nm}, \lambda \mathrm{em}=482 \mathrm{~nm})$. This spectral shift is used to differentiate bound and unbound ThT. $\beta$-Amyloid 1-40 (>95\%) was purchased from AnaSpec (Freemont, California) and stored at $-80^{\circ} \mathrm{C}$. All other reagents were of the highest available purity, purchased from Sigma-Aldrich (Oakville, Ontario), and used without further purification. All water used in the assays was micropore filtered and deionized. $\beta$-Amyloid 1-40 (1.0 mg) was pretreated in a $1.5 \mathrm{~mL}$ microfuge tube with $1 \mathrm{~mL}$ hexafluoro2-propanol (HFIP), and sonicated for five minutes to disassemble any pre-formed $\beta$-amyloid aggregates. Hexafluoro-2-propanol was removed using a stream of argon prior to dissolution of $\beta$-amyloid in Tris base ( $1 \mathrm{~mL}, 20 \mathrm{mM}, \mathrm{pH} 10)$ using vortex and 10 minutes sonication. The solution was then transferred to a $15 \mathrm{~mL}$ glass vial and diluted with a further $4.7 \mathrm{~mL}$ of the Tris base followed by adjusting to $\mathrm{pH} 7.4$ using concentrated $\mathrm{HCl}$ (aq) and filtered using a $0.2 \mu \mathrm{m}$ syringe filter.

The pretreated $\beta$-amyloid was diluted with an equal volume $(5.7 \mathrm{~mL})$ of $8 \mu \mathrm{M}$ ThT in Tris $(20 \mathrm{mM}, \mathrm{pH} 7.4,300 \mathrm{mM} \mathrm{NaCl})$ and $200 \mu \mathrm{L}$ aliquots were added to wells of a black polystyrene 96-well plate. $0.4 \mu \mathrm{L}$ of samples in dimethyl sulfoxide (DMSO) were added to each well. Each sample was performed in triplicate and DMSO alone served as a control to ensure that any observed anti-aggregant activity was accurately attributed to the samples being tested. Plates were covered with clear polystyrene lids and incubated in a Tecan Genios microplate reader at $37^{\circ} \mathrm{C}$ with

From the Toronto Western Research Institute (CH, DW), University Health Network; Division of Neurology (DW), Department of Medicine, University of Toronto; Treventis Corp. (YW, MT, DW), Toronto, Canada.

Received July 25, 2014. Final Revisions Submitted March 20, 2015.

Correspondence to: Donald F. Weaver, Toronto Western Research Institute,University of Toronto, 60 Leonard Ave.,Toronto, Ontario, M5T 2S8, Canada. E-Mail: dweaver@ uhnres.utoronto.ca 
fluorescence measurements recorded $(\lambda \mathrm{ex}=450 \mathrm{~nm}, \lambda \mathrm{em}=480 \mathrm{~nm})$ every 15 minutes after first being shaken at high intensity for 15 seconds and then allowed to settle for 10 seconds before each reading.

\section{ק-Amyloid Oligomerization Assay}

This assay, adapted from LeVine, ${ }^{6}$ was used to determine the ability of compounds to inhibit the oligomerization of biotinylated $\beta$-amyloid (1-42) utilizing an enzyme-linked immunosorbent assay, (ELISA) method on a NeutrAvidincoated plate. An ELISA plate (Costar 9018) was coated with $50 \mu \mathrm{L}$ of $1 \mu \mathrm{g} / \mathrm{mL}$ NeutrAvidin $^{\mathrm{TM}}$ (NA) in $10 \mathrm{mM}$ sodium phosphate buffer, $\mathrm{pH}$ 7.5. The plate was sealed with adhesive film and stored at $4{ }^{\circ} \mathrm{C}$ overnight prior to blocking for two hours at room temperature with $200 \mu \mathrm{L} /$ well of sodium phosphate buffer (20 mM, $150 \mathrm{mM} \mathrm{NaCl}, \mathrm{pH} 7.5)$ plus $0.1 \% \mathrm{v} / \mathrm{v}$ Tween 20. Then, $20 \mu \mathrm{L}$ of a $0.1 \mathrm{mg} / \mathrm{mL}$ solution of biotinylated $\beta$-amyloid was further diluted with $100 \mu \mathrm{L}$ HFIP and dried under a stream of nitrogen. Trifluoroacetic acid (TFA, $100 \mu \mathrm{L}$ ) was added to the tube and the sample was dissolved using a vortex mixer prior to drying under a stream of nitrogen. $100 \mu \mathrm{L}$ of HFIP was added and dried under a nitrogen stream to remove residual TFA. The biotinylated $\beta$-amyloid was then dissolved in $870 \mu \mathrm{L}$ of DMSO. $4.3 \mu \mathrm{L}$ of test compound dissolved in DMSO was added to each well of a 96-well polypropylene plate (Nunc 267245) and $430 \mu \mathrm{L}$ of $20 \mathrm{mM}$ sodium phosphate buffer, $150 \mathrm{mM} \mathrm{NaCl}, \mathrm{pH}$ 7.5). $2 \mu \mathrm{L}$ of the biotinylated $\beta$-amyloid solution was added to each well of a 96-well polypropylene plate (Costar 3365) followed by $100 \mu \mathrm{L}$ of diluted test compound. The plate was incubated for one hour at room temperature.

The NeutrAvidin-coated plate was warmed to room temperature and the blocking solution removed. $50 \mu \mathrm{L}$ of the biotinylated $\beta$-amyloid and test compound solution was added to each well and the plate was once again sealed and incubated for two hours with shaking at $150 \mathrm{rpm}$. A plate washer $(3 \times 30$ seconds, $200 \mu \mathrm{L} /$ well $)$ with $20 \mathrm{mM}$ Tris- $\mathrm{HCl}(150 \mathrm{mM}, \mathrm{pH} 7.5 / 0.1 \%$ Tween 20$)$ was used following incubation. Then, $50 \mu \mathrm{L}$ of Streptavidin-HRP $(1: 20,000)$ in sodium phosphate buffer $(20 \mathrm{mM}, 150 \mathrm{mM}$ $\mathrm{NaCl}, \mathrm{pH} 7.5+0.1 \%$ Tween 20 ) was added and sealed prior to incubation for one hour with shaking at $150 \mathrm{rpm}$. The plate was once again washed using $20 \mathrm{mM}$ Tris- $\mathrm{HCl}(150 \mathrm{mM}, \mathrm{pH} 7.5 / 0.1 \%$ Tween 20) $3 \mathrm{X} 30$ seconds, $200 \mu \mathrm{L} /$ well. $100 \mu \mathrm{L}$ of tetramethylbenzidine $\mathrm{TMB} / \mathrm{H}_{2} \mathrm{O}_{2}$ substrate solution was added to each well. Reaction was stopped after 5-10 minutes with the addition of $100 \mu \mathrm{L}$ of $1 \% \mathrm{v} / \mathrm{v}$ sulfuric acid prior to reading absorbance at $450 \mathrm{~nm}$ in a plate reader.

\section{Tau ThS Aggregation Assay}

The thioflavin $\mathrm{S}(\mathrm{ThS})$ assay is a fluorescence assay based assay used to visualize the aggregation of tau protein; $\mathrm{ThS}$ is a benzothiazole dye with a high affinity for proteins containing high $\beta$-sheet content. The assay protocol is analogous to the ThT assay used to examine the aggregation of $\beta$-amyloid except for the requirement of an inducer (heparin) to initiate tau aggregation. Full-length human tau, 2N4R isoform, was purchased from rPeptide (Bogart, GA). Each experiment consisted of a single replicate and was repeated four times.
A $4 \mu \mathrm{M}$ solution of tau in $5 \mu \mathrm{M}$ ThS in Tris $(50 \mathrm{mM}$, pH 7.4, $0.01 \mathrm{mg} / \mathrm{mL}$ heparin, $1 \mathrm{mM}$ DTT, $50 \mu \mathrm{M} \mathrm{NaN}$ ) was prepared fresh for each experiment and $200 \mu \mathrm{L}$ aliquots were added to wells of a black polystyrene 96 -well plate. $0.4 \mu \mathrm{L}$ of samples in DMSO were added to each well. Each sample was performed in triplicate and DMSO alone served as a control to ensure that any observed anti-aggregant activity was accurately attributed to the samples being tested. Plates were covered with clear polystyrene lids and incubated in a Tecan Genios microplate reader at $37^{\circ} \mathrm{C}$ with fluorescence measurements recorded $(\lambda \mathrm{ex}=450 \mathrm{~nm}$, $\lambda \mathrm{em}=480 \mathrm{~nm}$ ) every 15 minutes.

\section{Results}

Three separate extracts of maple syrup were sequentially isolated: Extract $\mathbf{A}$ - ethyl acetate soluble; Extract $\mathbf{B}-n$-butanol soluble, methanol insoluble; Extract $\mathbf{C}-n$-butanol soluble, methanol soluble. Each of these three extracts was then evaluated for anti-protein misfolding activity in three separate assays: [i] A $\beta$ anti-oligomerization assay, [ii] $\mathrm{A} \beta$ anti-aggregation assay, and [iii] tau anti-aggregation assay. The use of thioflavin $\mathrm{T}$ and thioflavin $\mathrm{S}$ allowed for the dynamic measurement of aggregation such as lag time, initial rate of beta-sheet formation and equilibrium levels. A clear distinction in activity between the three extracted fractions from maple syrup was noted, with the ethyl acetate soluble fraction (A) demonstrating significant $\mathrm{A} \beta$ anti-oligomeric and $\mathrm{A} \beta$ anti-aggregant activities as well as the ability to prevent tau aggregation. Extract $\mathbf{A}$ was shown to inhibit A $\beta$ aggregation by $47 \%$ at $30 \mathrm{ppm}$, and demonstrated concentration-dependent activity in the ThT assay (Figures 1,2). Extracts $\mathbf{B}$ and $\mathbf{C}$ did not exhibit any significant activity, and there was no detectable effect of compound concentration on $\mathrm{A} \beta$ aggregation. This trend was also demonstrated in the $\mathrm{A} \beta$ oligomerization assay with $\mathbf{A}$ demonstrating significant activity, while both Extracts $\mathbf{B}$ and $\mathbf{C}$ showed no significant difference from the control ( $p=0.05$, two-tailed, Figures 3 and 4). In addition, the thioflavin $\mathrm{S}$ assay was employed to determine whether extract $\mathbf{A}$ would also prevent aggregation of tau; extract $\mathbf{A}$ was shown to inhibit the aggregation of tau by $41 \%$ at a concentration

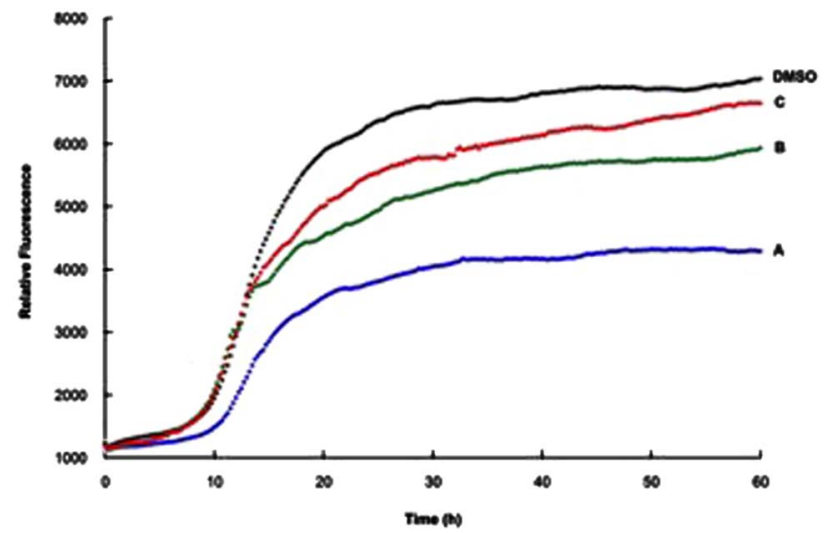

Figure 1: Thioflavin $T$ assay results. $\beta$-Amyloid was incubated with $100 \mu M$ of each maple syrup extract, A - ethyl acetate soluble; $B$ - butanol soluble, methanol insoluble; $C$ - butanol and methanol soluble. DMSO was used as a control compound. 


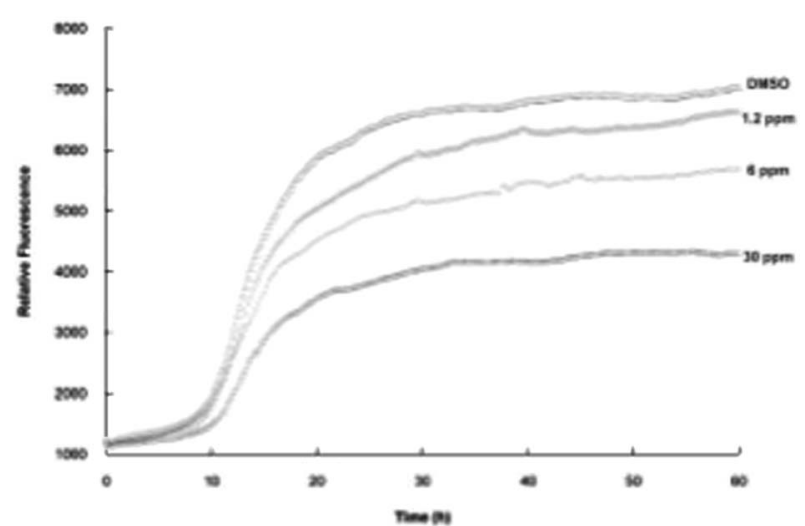

Figure 2: Thioflavin $T$ assay results. $\beta$-Amyloid was incubated with varying concentrations of the ethyl acetate extract of maple syurp $(C)$. DMSO was used as a control.

of $30 \mathrm{ppm}$ (Figure 5). Extracts $\mathbf{B}$ and $\mathbf{C}$ had no activity in the ThS aggregation assay.

Although Extract $\mathrm{A}$ has significant anti-protein misfolding activities, it is difficult to identify which exact compound(s) imparts these activities. Li et al. have identified a wide variety of compounds including lignans, polyphenols and a phenylpropanoids within ethyl acetate extracts of maple syrup. ${ }^{4}$ Moreover, it is possible that a single component of the extract is not responsible for the observed biological activities, and that a combination of components leads to the observed results. Nonetheless, the results of this study do indicate that the ethyl acetate extract of maple syrup has significant anti-protein misfolding activity which is within the range of other natural product polyphenols. For example, the IC-50 for inhibition of A $\beta$ oligomerization by maple syrup Extract $\mathrm{A}$ was $6.6 \mathrm{ppm}$, which is comparable to a range of other polyphenols, including resveratrol (red wine extract)

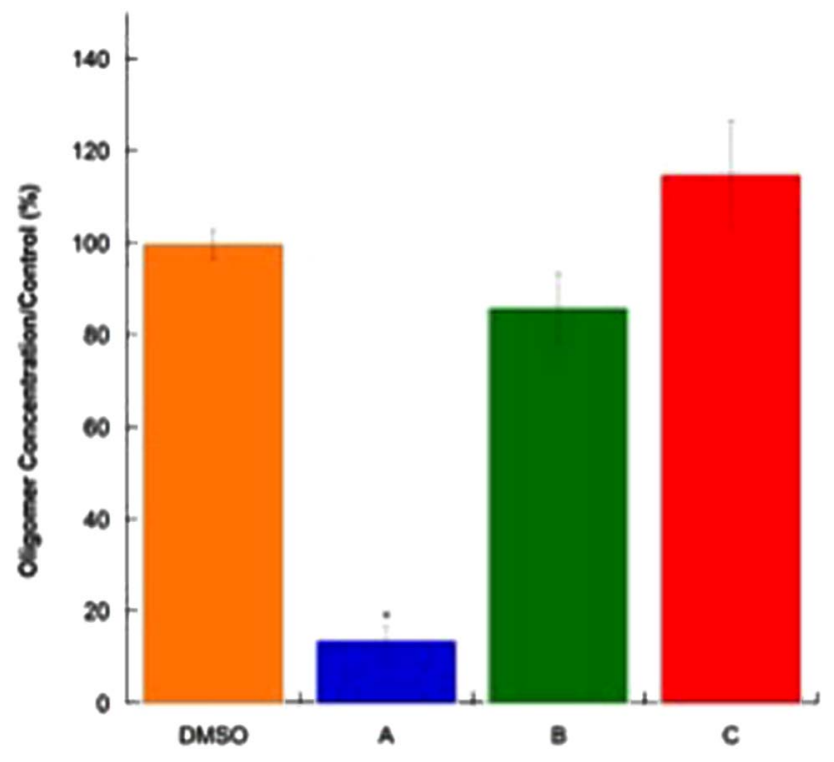

Figure 3: $\beta$-Amyloid oligomerization assay results for maple syrup extracts $(100 \mu M)$. Extract $A$, is shown to cause a significant decrease in oligomer formation as indicated by * $(p=0.05)$. DMSO was the control compound used.

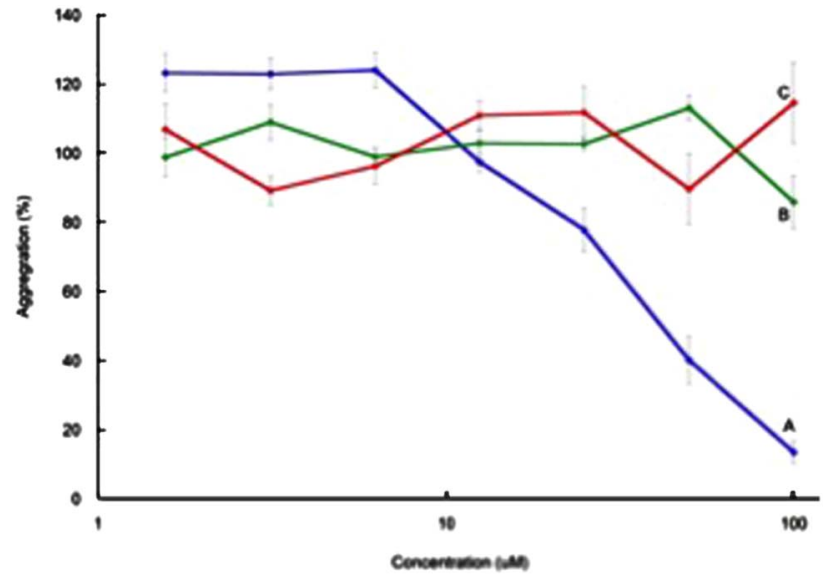

Figure 4: $\beta$-Amyloid oligomerization results. $\beta$-Amyloid was incubated with $100 \mu M$ of each maple syrup extract, $A$ - ethyl acetate soluble; $B-$ butanol soluble, methanol insoluble; $C$ - butanol an $\mu d$; methanol soluble. DMSO was used as a control compound.

and (-)-epigallocatechin-3-gallate (green tea extract), which have IC-50s in the $12-15 \mu \mathrm{M}$ range. Investigations into the ability of these compounds to cross the blood brain barrier must be performed in order to estimate the required intake of maple syrup needed to have a therapeutic effect along with more advanced in vivo experiments.

\section{CONCLUSIONS}

This study provides a uniquely Canadian addition to the literature pertaining to the potential anti-Alzheimer's benefits of natural product polyphenols. Through a series of in vitro experiments we were able to identify the potential ability of an ethyl acetate extract of maple syrup to reduce $\beta$-amyloid and tau aggregation, two pathological hallmarks of Alzheimer's disease. It is still unknown whether the compounds and/or their metabolites are able to cross the blood brain barrier, with more advanced in vivo models being required to further understand the potential activities. While we are currently unable to identify a specific component of the extract responsible for this activity, the

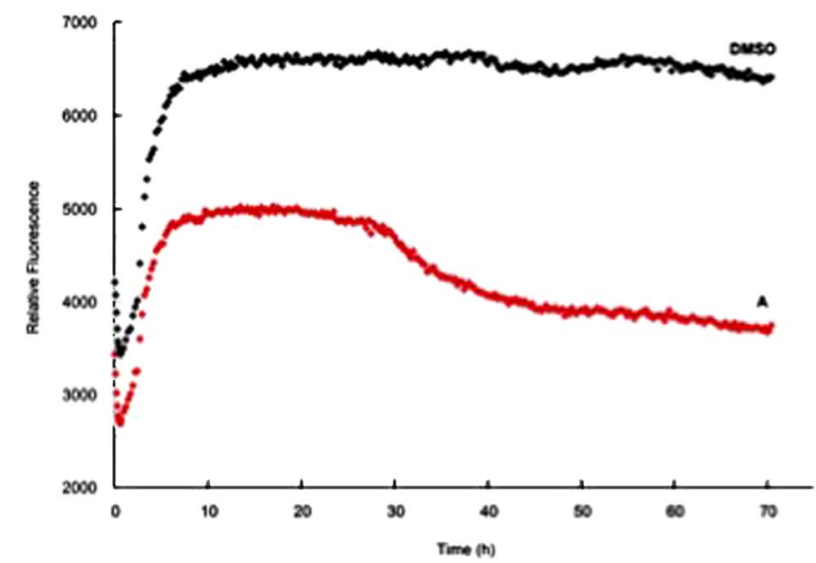

Figure 5: Thioflavin $S$ assay results. $\beta$-amyloid was incubated with $100 \mu \mathrm{M}$ of the ethyl acetate soluble extract of maple syrup (A). DMSO was used as a control compound. 
antiaggregation properties of maple syrup are analogous to those of other natural products extracted from red wine or green tea. Further work is required to ascertain if maple syrup derived natural products could be a starting point in the design and development of new therapeutics for AD.

\section{Disclosures}

Donald Weaver and Cassandra Hawco have nothing to disclose. Marcia Taylor has the following disclosure: Treventis Corp, Director, Salary, Stock options. YanFei Wang has the following disclosure: Treventis Corp, Director, Salary, Stock options.

\section{REFERENCES}

1. Statistics Canada. Table 001-0008 - Production and farm value of maple products, annual, CANSIM (database) (accessed: 2014-06-23).
2. Legault J, Girard-Lalancette K, Grenon C, Dussault C, Pichette A. Antioxidant activity, inhibition of nitric oxide overproduction, and in vitro antiproliferative effect of maple sap and syrup from Acer saccharum. J Med Food. 2010;13:460-8

3. Gonzalez-Sarrias A, Li L, Seeram NP. Anticancer effects of maple syrup phenolics and extracts on proliferation, apoptosis, and cell cycle arrest of human colon cells. J Funct Foods. 2012:4:185-96.

4. Li L, Seeram NP. Further investigation into maple syrup yields three new lignans, a new phenylpropanoid, and twenty-six other phytochemicals. J Agric Food Chem. 2011;59:7708-16.

5. Li L, Seeram NP. Maple syrup phytochemicals include lignans, coumarins, a stilbene and other previously unreported antioxidant phenolic compounds. J Agric Food Chem. 2010;58:11673-9.

6. LeVine H. Biotin-avidin interaction-based screening assay for Alzheimer's $\beta$-peptide oligomer inhibitors. Anal Biochem. 2006;356:265-72. 